\title{
MicroRNA-451 regulates activating transcription factor 2 expression and inhibits liver cancer cell migration
}

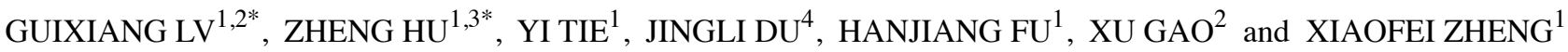 \\ ${ }^{1}$ Beijing Institute of Radiation Medicine, Beijing 100850; ${ }^{2}$ Department of Biochemistry and Molecular Biology, \\ Harbin Medical University, Harbin, Heilongjiang 150086; ${ }^{3}$ Laboratory of Sono- and Photo-theranostic Technologies, \\ Harbin Institute of Technologies, Harbin, Heilongjiang 150080; ${ }^{4}$ Department of Pathology, \\ General Hospital of PLA, Beijing 100853, P.R. China
}

Received April 17, 2014; Accepted June 11, 2014

DOI: $10.3892 /$ or.2014.3296

\begin{abstract}
Accumulating evidence suggests that microRNAs (miRNAs) can function as oncogenes or as tumor suppressor genes depending on the tissue type or target. Therefore, clarification of the specific roles of miRNAs is vital for the diagnosis and treatment of cancer. In the present study, miR-451 was found to be downregulated in hepatocellular carcinoma (HCC) tissues when compared to that in adjacent tissues. Functional analysis showed that, in vitro, miR-451 inhibited the migration of hepatoma cell lines HepG2 and SK-Hep-1. Further investigation of the molecular mechanisms identified activating transcription factor 2 (ATF2) as a target of miR-451. miR-451 inhibited ATF2 expression by binding to the 3'UTR. An in vivo assay revealed a significant negative correlation between miR-451 and ATF2 in liver cancer tissues. According to previous findings reported in the literature, the opposing functions of ATF2 are related to its subcellular localization. In the nucleus, ATF2 displays oncogenic activities in melanoma. In the present study, ATF2 exhibited a higher expression level in the nucleus in tumoral tissues of HCC as detected by immunohistochemistry. In conclusion, in this study, we identified a potential target of miR-451, ATF2, and revealed a novel role of miR-451 in the inhibition of the migratory ability of hepatoma cell lines.
\end{abstract}

Correspondence to: Professor Xiaofei Zheng, Beijing Institute of Radiation Medicine, 27 Taiping Road, Beijing 100850, P.R. China E-mail: xfzheng100@126.com

Professor Xu Gao, Department of Biochemistry and Molecular Biology, Harbin Medical University, Harbin, Heilongjiang 150086, P.R. China

E-mail: gaoxu6712@163.com

*Contributed equally

Key words: miR-451, liver cancer, migration, activating transcription factor 2

\section{Introduction}

Hepatocellular carcinoma (HCC) is one of the most common cancer types in both genders. Due to its high mortality, HCC is the third most common cause of cancer-related death (1). Because of a high incidence of recurrence after initial treatment and high migratory capacity, patients with HCC have a poor prognosis with only a 5-year survival rate of $10 \%$ from initial diagnosis (2). The majority of HCC cases occur in developing countries resulting in a serious economic and social burden that cannot be ignored.

Viral infections, abnormal metabolic alteration, epigenetic or genetic changes in liver tissues may finally lead to HCC. During tumor genesis or progression, the expression levels of a series of genes are dysregulated such as oncogenes or tumor suppressors. In addition, the location of specific proteins is also related to the behavior of cancer cells. Notably, the expression levels of almost $60 \%$ of genes in mammalian cells are regulated by microRNAs (miRNAs). An increased understanding concerning the molecular mechanisms involved in $\mathrm{HCC}$ will facilitate diagnosis and effective treatment.

miRNAs are small conserved noncoding RNAs of approximately 22 nucleotides that function to fine-tune gene expression by targeting preferential sequences in the 3' untranslated region (UTR) of mRNAs (3). The roles of miRNAs in cancer initiation or progression depend on their targets or the specific tissue origin (4). Several miRNAs have been shown to be abnormally expressed and to be related to the prognosis (5), metastasis (6) and therapeutic response of HCC (7) since the beginning of studies on the roles of miRNAs in HCC. Moreover, as an important application that is gradually being explored, various circulating miRNAs can be prognostic biomarkers for $\operatorname{HCC}(8,9)$.

In our previous study, an miRNA expression profile was carried out in tumor tissues and adjacent non-tumoral liver tissues from patients with HCC. miR-451 was found to have lower expression in the tumor tissues. Yet, the function of miR-451 remains unknown. In the present study, the result from the microassay was further confirmed by qRT-PCR that miR-451 was downregulated in 24 of the 26 HCC tumor tissues. ATF2, a key component of activator protein 1 (AP1), was found to be a target of miR-451 by sequence analysis and 
luciferase reporter assay. In contrast to miR-451, ATF2 was increased in tumor tissues where it was mainly localized in the nucleus. Further study showed that miR-451 inhibited the migration of HepG 2 and SK-Hep-1 cells. These data revealed the potential function of miR-451 and a new pathway for the regulation of ATF2 in $\mathrm{HCC}$.

\section{Materials and methods}

Clinical tissue specimens. Twenty-six patients with HCCs at the General Hospital of PLA (Beijing, China) were enrolled. No patient in the present study received preoperative adjuvant therapy prior to surgery. The fresh tumoral and peritumoral tissue specimens for RNA extraction were frozen in liquid nitrogen immediately after surgery. The clinical features of the patients are characterized in Table I. Informed consent was obtained from the patients, and this study was approved by the Medical Ethics Committee of the General Hospital of PLA.

Cell lines and cultures. Human liver cancer cell lines HepG2 and SK-Hep-1 were cultured in Dulbecco's modified Eagle's medium (DMEM) (Gibco-BRL, Grand Island, NY, USA) supplemented with $10 \%$ fetal bovine serum (FBS) (Hyclone, Thermo Scientific, Rockford, IL, USA), $100 \mathrm{U} / \mathrm{ml}$ penicillin and $100 \mu \mathrm{g} / \mathrm{ml}$ streptomycin in a $5 \% \mathrm{CO}_{2}$ and $95 \%$ air incubator at $37^{\circ} \mathrm{C}$.

Plasmid construction and transfection. For studying the target effect of miR-451 on ATF2, the 3'UTR segment of ATF2 was subcloned into a modified pGL3 control vector (10) immediately downstream of the luciferase gene's stop codon after PCR amplification of genomic DNA with the primers: pGL3-ATF2-3'UTR (F: 5'-AGTTCTAGATTAAAAACCT GCAGTACAACAGT-3' and R: 5'-AGTCATATGTTTTCA GTAACACCCCCATTTAT-3'), and the pGL3-ATF23'UTRmut vector was generated with specific primer substitutions at the miRNA complementary sites using PCR. Wild-type or mutant inserts were confirmed by sequencing. DNA transfection was performed using JetPrime ${ }^{\circledR}$ transfection reagent (Polyplus, France) according to the manufacturer's protocol.

miRNA mimics, siRNAs and transfection. miR-451 mimics (guide, 5'-AAACCGUUACCAUUACUGAGUUU-3' and passenger, 5'-ACUCAGUAAUGGUAACGGUUUUU-3'), siRNAs against ATF2 (sense, 5'-GUUGGCGAGUCCAUUU GAGTT-3' and antisense, 5'-CUCAAAUGGACUCGCCAA CTT-3') and negative control RNA duplex (sense, 5'-GGCUAC GUCCAGGAGCGCACC-3' and antisense, 5'-UGCGCUCCU GGACGUAGCCTT-3') were synthesized by GenePharma (Shanghai, China). Cells grown in 6-well plates were transfected with siATF2 (20 nM) or miR-451 mimics (20 nM) using Interferin ${ }^{\circledR}$ reagent (Polyplus) according to the manufacturer's protocol. Total-RNA and protein were prepared at $48 \mathrm{~h}$ and $72 \mathrm{~h}$, respectively, after transfection.

Real-time RT-PCR for detection of mRNA and miRNA. Total-RNA from the tissues or the cultured cells was isolated using TRI ${ }^{\circledR}$ reagent (Sigma-Aldrich, St. Louis, MO, USA). For mRNA detection, cDNA was synthesized from total-RNA using Oligo $(\mathrm{dT})_{18}$ primer and ImProm-II ${ }^{\mathrm{TM}}$ reverse transcriptase system (Promega, Madison, WI, USA) according to the manufacturer's protocol. The real-time PCR was performed by using SYBR Premix Ex Taq (Takara Bio, Dalian, China). Primers used for mRNA detection were: ATF2 (F: 5'-CTCCAG CTCACACAACTCCA-3' and R: 5'-TGTTTCAGCTGTGC CAC TTC-3') and GAPDH (F: 5'-TCAGTGGTGGACCTG ACCTG3' and R: 5'-TGCTGTAGCCAAATTCGTTG-3'). Data were normalized to the GAPDH mRNA level.

For miRNA detection, poly(A)-tailed RNA was used in real-time RT-PCR (11). In brief, $10 \mu \mathrm{g}$ total-RNA, $1 \mathrm{mM}$ rATP, $5 \mu \mathrm{l} 10 \mathrm{X}$ poly(A) polymerase reaction buffer and 2 units poly(A) polymerase (NEB, UK) were mixed in a $50-\mu 1$ reaction system and incubated at $37^{\circ} \mathrm{C}$ for $60 \mathrm{~min}$ according to the manufacturer. Then the poly(A)-tailed total-RNA was recovered using water-saturated phenol/chloroform extraction and precipitated with ethanol. The $1 \mu \mathrm{g}$ poly(A)-tailed total-RNA was reverse transcribed using $1 \mu \mathrm{g}$ miR-RT primer (5'-GCGA GCACAGAATTAATACGACTCACTATAGG(t)18VN-3') and $1 \mu 1 \mathrm{ImProm}$ reverse transcriptase (Promega) according to the manufacturer's protocol. Real-time PCR was performed using SYBR-Green PCR Mix kit (Qiagen, Hilden, Germany) according to the manufacturer. Primers used in qPCR were: miR-451 (F: 5'-AAACCGTTACCATTACTGAGTT-3' and R: 5'-GCGAGCACAGAATTAATACGAC-3') and U6 snRNA (F: 5'-CTCGCTTCGGCAGCACA-3' and R: 5'-AACGCT TCACGAATTTGCGT-3'. The U6 snRNA level was used for normalization.

Western blot analysis. Protein extracts were prepared in a modified RIPA buffer [50 mM Tris- $\mathrm{HCl}$ pH 7.4, $150 \mathrm{mM} \mathrm{NaCl}$, $1 \%$ (v/v) NP-40, $0.25 \%$ (w/v) Na deoxycholate, $0.5 \%$ (w/v) SDS and $1 \mathrm{X}$ protease inhibitor cocktail (Complete Mini, Roche, Switzerland)]. Equal amounts of proteins were separated by $10 \%$ SDS-PAGE and were electrotransferred onto Immobilon Hybond-C membranes (Amersham Biosciences, Piscataway, NJ, USA). Primary antibodies against ATF2 (1489; Epitomics) and $\beta$-actin (sc-47778; Santa Cruz) were used and detected with SuperSignal ${ }^{\circledR}$ West Pico chemiluminescent substrate (Thermo Scientific).

Luciferase reporter assay. HepG2 cells in 24-well plate were transfected with mixtures containing $100 \mathrm{ng}$ firefly luciferase reporter plasmid with the ATF2 3'UTR (named pGL3-ATF2-3'UTR) or ATF2 3'UTR mutant (named pGL3-ATF2-3'UTRmut) sequence, miR-451 mimics (20 nM), and $1 \mathrm{ng}$ pRL-TK (a normalization control). Luciferase activities were measured using a dual-luciferase reporter assay system (Promega) at $48 \mathrm{~h}$ after transfection.

Cell migration assay. HepG2 and SK-Hep-1 cells cultured in 6-well plates were transfected using miR-451 mimics or negative control (NC). Cells were serum-starved for $18 \mathrm{~h}$ in DMEM containing $0.1 \%$ FBS from $30 \mathrm{~h}$ after transfection. Then the starved cells were trypsinized and resuspended in DMEM containing $0.1 \% \mathrm{FBS}$, and $2 \times 10^{5}$ cells were added to the upper chamber with a $6.5-\mathrm{mm}$ diameter, $8-\mu \mathrm{m}$ pore size membrane (Corning), while the DMEM containing 5\% FBS was placed in the lower compartment of the chamber. After incubation at $37^{\circ} \mathrm{C}$ for $12 \mathrm{~h}$, cells on the supine surface of the membrane 
Table I. Clinical features of the patients with HCC.

\begin{tabular}{|c|c|c|c|c|c|c|c|}
\hline Patient no. & $\begin{array}{c}\text { Age } \\
\text { (years) }\end{array}$ & Gender & $\begin{array}{c}\text { Tumor size } \\
(\mathrm{cm} \mathrm{x} \mathrm{cm} \mathrm{x} \mathrm{cm)}\end{array}$ & $\mathrm{HbsAg}$ & $\mathrm{HCV}-\mathrm{Ab}$ & $\operatorname{AFP}(\mu \mathrm{g} / 1)$ & Cirrhosis \\
\hline 1 & 66 & M & $4 \times 3 \times 2$ & $\mathrm{P}$ & $\mathrm{N}$ & 38.88 & Yes \\
\hline 2 & 49 & M & $11 \times 7.5 \times 7$ & $\mathrm{P}$ & $\mathrm{N}$ & 1.26 & No \\
\hline 3 & 43 & $\mathrm{M}$ & $6.5 \times 6 \times 2.5$ & $\mathrm{P}$ & $\mathrm{N}$ & 3.76 & Yes \\
\hline 4 & 67 & M & $7.5 \times 6.5 \times 4$ & $\mathrm{~N}$ & $\mathrm{~N}$ & $14,284.00$ & Yes \\
\hline 5 & 58 & M & $3 \times 2 \times 2$ & $\mathrm{~N}$ & $\mathrm{~N}$ & $1,139.00$ & Yes \\
\hline 6 & 70 & $\mathrm{~F}$ & $7 \times 7 \times 6$ & $\mathrm{~N}$ & $\mathrm{~N}$ & 157.10 & No \\
\hline 7 & 70 & M & $9 \times 8.5 \times 6$ & $\mathrm{P}$ & $\mathrm{N}$ & 3.69 & Yes \\
\hline 8 & 55 & M & $5 \times 4 \times 3$ & $\mathrm{~N}$ & $\mathrm{~N}$ & $6,044.00$ & No \\
\hline 9 & 67 & M & $6 \times 2 \times 2$ & $\mathrm{~N}$ & $\mathrm{~N}$ & 24.86 & No \\
\hline 10 & 72 & $\mathrm{~F}$ & $4 \times 3 \times 3$ & $\mathrm{P}$ & $\mathrm{N}$ & $5,830.00$ & Yes \\
\hline 11 & 50 & $\mathrm{~F}$ & $6 \times 6 \times 3.5$ & $\mathrm{P}$ & $\mathrm{N}$ & 5.34 & Yes \\
\hline 12 & 49 & M & $9 \times 6 \times 3$ & $\mathrm{P}$ & $\mathrm{N}$ & $20,000.00$ & No \\
\hline 13 & 25 & M & $10 \times 7.5 \times 7$ & $\mathrm{~N}$ & $\mathrm{~N}$ & $20,000.00$ & No \\
\hline 14 & 48 & $\mathrm{M}$ & $18 \times 15 \times 8$ & $\mathrm{P}$ & $\mathrm{N}$ & 968.50 & Yes \\
\hline 15 & 52 & M & $6.5 \times 6 \times 3$ & $\mathrm{P}$ & $\mathrm{N}$ & 468.50 & Yes \\
\hline 16 & 40 & M & $7 \times 5 \times 3$ & $\mathrm{P}$ & $\mathrm{N}$ & 210.20 & Yes \\
\hline 17 & 70 & $\mathrm{~F}$ & $5.5 \times 5 \times 4$ & $\mathrm{P}$ & $\mathrm{N}$ & 862.90 & Yes \\
\hline 18 & 46 & M & $7 \times 6 \times 5$ & $\mathrm{P}$ & $\mathrm{N}$ & 7.25 & Yes \\
\hline 19 & 56 & M & $2.5 \times 2 \times 2$ & $\mathrm{P}$ & $\mathrm{N}$ & 4.09 & No \\
\hline 20 & 60 & M & $2.5 \times 2 \times 2$ & $\mathrm{P}$ & $\mathrm{N}$ & 8.25 & No \\
\hline 21 & 58 & $\mathrm{~F}$ & $19 \times 15 \times 13$ & $\mathrm{~N}$ & $\mathrm{~N}$ & 3.81 & No \\
\hline 22 & 42 & M & $4 \times 4 \times 3.5$ & $\mathrm{P}$ & $\mathrm{N}$ & 167.00 & Yes \\
\hline 23 & 47 & $\mathrm{~F}$ & $3 \times 3 \times 3$ & $\mathrm{P}$ & $\mathrm{N}$ & 8.92 & Yes \\
\hline 24 & 60 & $\mathrm{~F}$ & $14 \times 11 \times 8$ & $\mathrm{P}$ & $\mathrm{N}$ & 1.41 & No \\
\hline 25 & 62 & F & $4 \times 3 \times 3$ & $\mathrm{~N}$ & $\mathrm{~N}$ & 2.42 & No \\
\hline 26 & 49 & M & $11 \times 7 \times 4.8$ & $\mathrm{P}$ & $\mathrm{N}$ & 21.06 & No \\
\hline
\end{tabular}

F, female; M, male; P, positive; N, negative; HbsAg, hepatitis B surface antigen; HCV-Ab, hepatitis C virus antibody; AFP, $\alpha$-fetoprotein.

were removed carefully with a cotton swab, and then the filters were fixed with $95 \%$ ethanol for $30 \mathrm{~min}$, stained with $0.2 \%$ crystal violet solution for $30 \mathrm{~min}$, and finally observed under a microscope.

Immunohistochemical analysis. For detection of ATF2 expression in tissues, the sections $(4-\mu \mathrm{m})$ were prepared and subjected to deparaffinized in xylol and rehydrated in a graded alcohol series. Antigen retrieval was performed by boiling tissue sections in EDTA buffer ( $\mathrm{pH}$ 8.0) for $5 \mathrm{~min}$ at $100^{\circ} \mathrm{C}$ in a pressure cooker. Endogenous peroxidase activity was blocked with hydrogen peroxidase $(0.3 \%)$, and nonspecific proteins were blocked with $5 \%$ BSA. Incubation with the primary antibody against ATF2 (1:200) was carried out overnight at $4^{\circ} \mathrm{C}$, followed by the biotinylated secondary antibody and detection with diaminobenzidine (Dako) and counterstained with Mayer's hematoxylin. For each tissue specimen, ATF2 expression was scored on a scale of $0-3$ according to the extent (0-100\%) and intensity (strong, 3; moderate, 2; and weak, 1) which was evaluated by an observer in a blinded manner, and the average was calculated.
Statistical analysis. All values are reported as means \pm SD. Differences were estimated by the two-tailed Student's t-test using Excel software. Spearman's rank correlation was carried out to evaluate the relationship between miR-451 and ATF2 expression. $\mathrm{P}<0.05$ was considered to indicate a statistically significant difference.

\section{Results}

miR-451 is underexpressed in primary HCC lesions. The clinical features of the HCC patients in this study are described in Table I. Twenty-six pairs of fresh tumoral and peritumoral tissues were employed for detecting the expression of miR-451 by real-time RT-PCR. As shown in Fig. 1, miR-451 was underexpressed (1.3- to 315 -fold) in $92 \%$ of the tumors ( 24 of 26 patients) compared to the matching peritumoral tissues. The results suggest that miR-451 could be involved in most processes of HCC.

miR-451 interacts with the 3'UTR of ATF2 mRNA. miRNAs usually carry out their function by binding to the 3'UTR and 


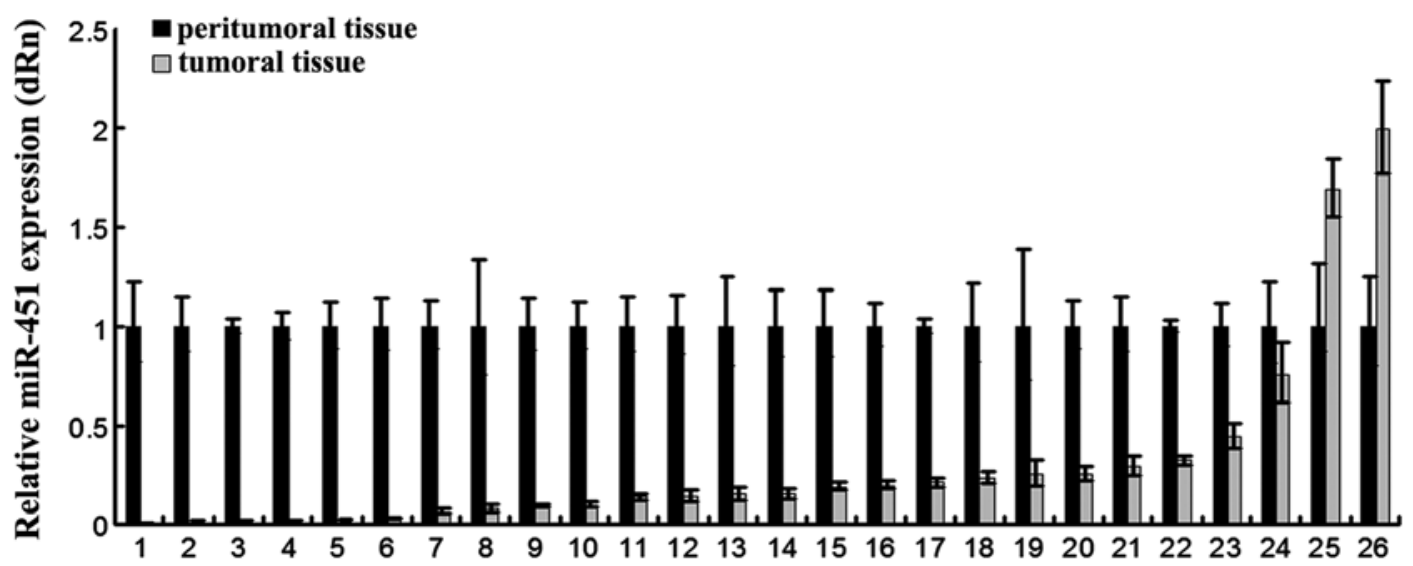

Figure 1. Relative miR-451 expression in tumoral and peritumoral tissues in a set of independent samples.

A

H.sapiens ATF2 3' UTR
has-miR-451

H.sapiens ATF2 3' UTR

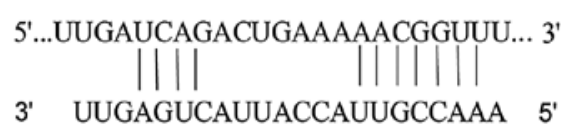

5'...UUGAUCAGACUGAAAUUGCCAAU...3'

\|\|

3' UUGAGUCAUUACCAUUGCCAAA 5
has-miR-451

2 3' UTRmut

B

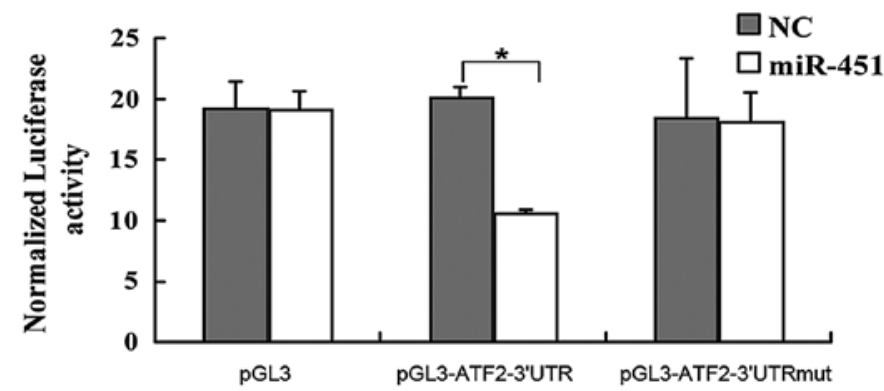

Figure 2. miR-451 interacts with the 3'UTR of ATF2 mRNA. (A) The binding site of miR-451 on ATF2 3'UTR was predicted by TargetScan. The short line shows the potential binding sequence. The ATF2 3'UTRmut exhibits change at the seed sequence of the binding site while the others are identical to the wild type. (B) miR-451 mimics or the negative control was co-transfected with the pGL3-ATF2-3'UTR or pGL3-ATF2-3'UTRmut reporter plasmid into HepG2 cells. Luciferase activity was normalized to another co-transfected Renilla expression plasmid. Results are shown as the mean relative luciferase activity \pm SD for three independent experiments. ${ }^{*} \mathrm{P}<0.05$.

inhibiting the expression of target mRNAs. By bioinformatic analysis using TargetScan (www.targetscan.org), we found that the 3'UTR of ATF2 contained a putative binding site for miR-451 (Fig. 2A). To investigate whether ATF2 is a direct target of miR-451, the human ATF2 wild-type 3'UTR and mutant-type 3 'UTR containing the mutant sequence at the miR-451 binding site were subcloned into the pGL3 vector separately after the firefly luciferase opening reading frame (ORF). These vectors were cotransfected with miR-451 mimics or the negative control (NC) RNA duplex into the HepG2 cells, respectively. The dual-luciferase reporter assay showed that miR-451 significantly decreased the relative luciferase activity $(\sim 37 \%)$ of the reporter containing the wildtype $3^{\prime}$ UTR as compared to the negative control RNA but no decrease in luciferase activity of the reporter was detected when the putative miR-451 binding site was mutated (Fig. 2B). This result suggests that ATF2 is a direct target of miR-451.
miR-451 suppresses the expression of ATF2. Inhibition of expression by miRNAs may be mediated by degrading mRNAs $(12,13)$ or impeding translation without affecting the stability of mRNAs (14). To ascertain whether miR-451 inhibits endogenous ATF2 expression, miR-451 mimics were transfected into HepG2 and SK-Hep-1 cells, respectively. Total-RNA and protein were extracted at $48 \mathrm{~h}$ and $72 \mathrm{~h}$ after transfection. Results from RT-PCR and western blot analysis showed that ATF2 mRNA (Fig. 3A and C) and protein (Fig. 3B and D) were significantly reduced after transfection of miR-451 mimics as compared to the negative control. The results revealed that miR-451 suppresses ATF2 expression at the post-transcriptional level by degrading its mRNA.

A significant negative correlation between $m i R-451$ and ATF2 expression is observed in human HCC. To determine the relationship between the expression of miR-451 and 
A

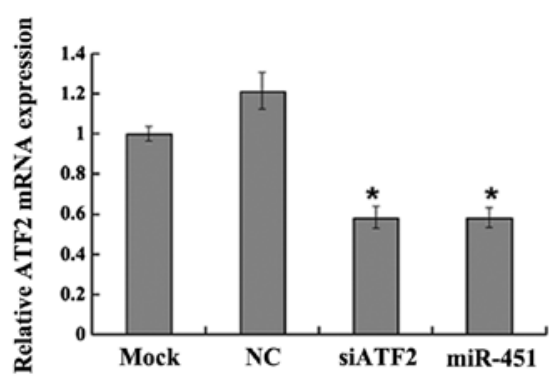

C

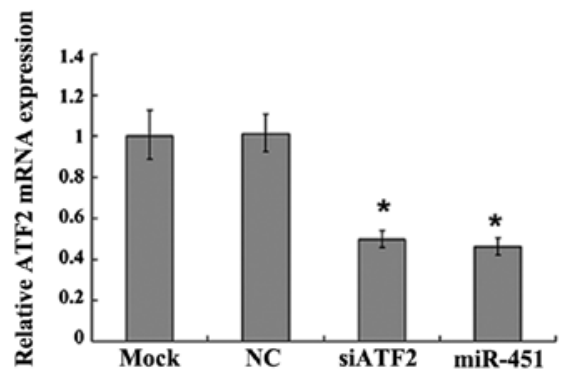

B

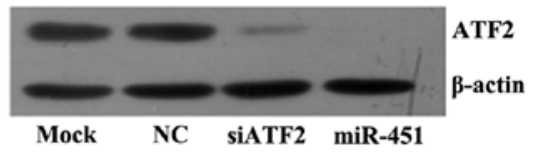

D

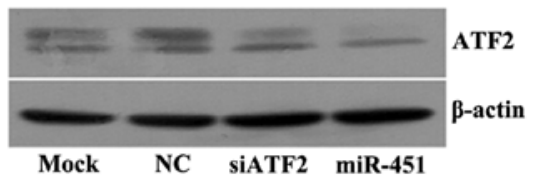

Figure 3. miR-451 inhibits ATF2 expression in hepatoma cell lines. SK-Hep-1 and HepG2 cells were transfected with miR-451 mimics, siRNA against ATF2 or the negative control (NC). (A and C) ATF2 mRNA expression in SK-Hep-1 and HepG2 cells at $48 \mathrm{~h}$ after transfection was detected by qRT-PCR. The expression level was normalized to GAPDH. "P<0.05 vs. the control. (B and D) ATF2 protein expression in SK-Hep-1 and HepG2 cells at $72 \mathrm{~h}$ after transfection was detected by western blotting; $\beta$-actin was used as a loading control.

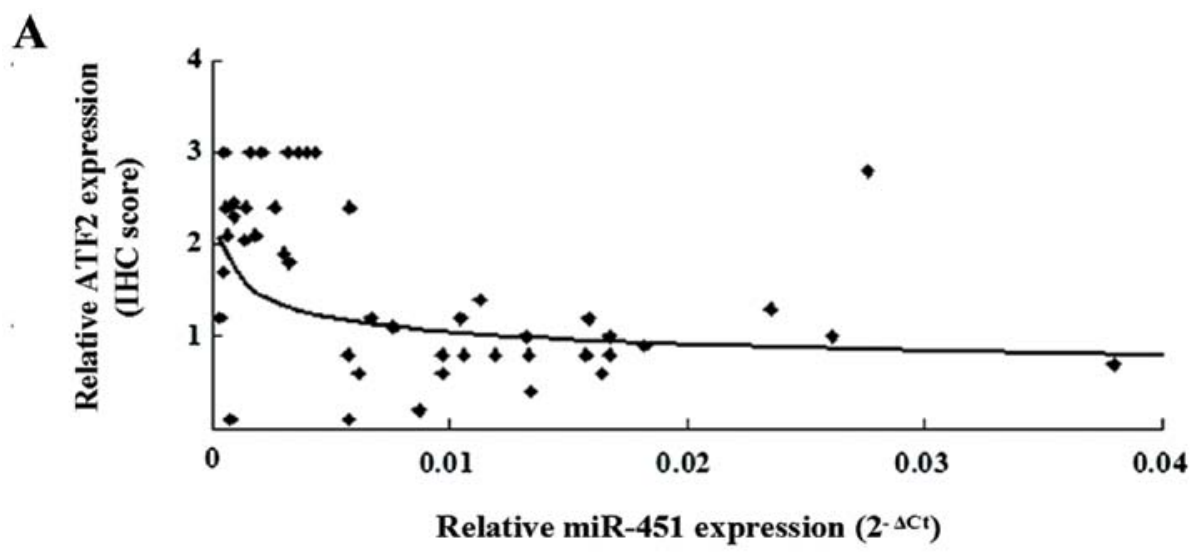

B

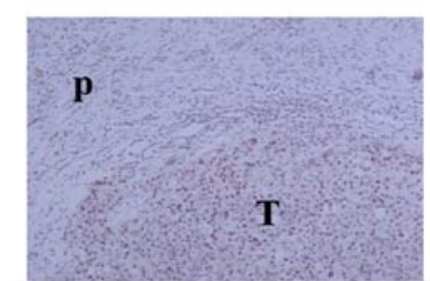

Tumoral and Peritumoral tissue $(\times 100)$

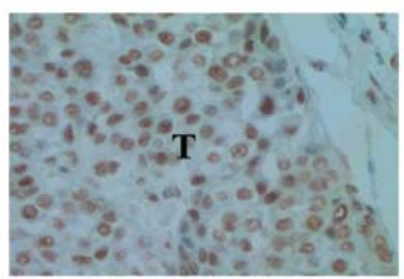

Tumoral tissue $(\times 400)$

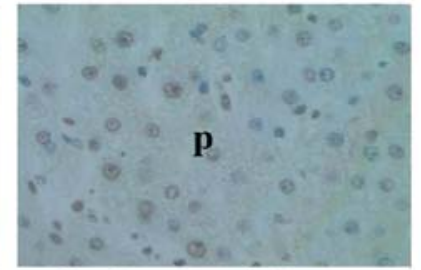

Peritumoral tissue $(\times 400)$

Figure 4. miR-451 and ATF2 exhibit an obvious inverse relationship in HCC tissues. (A) Relative miR-451 expression is represented by the $\Delta$ Ct method with snRNA U6 as endogenous reference. Data show miR-451 relative expression as $2^{-\Delta \mathrm{Ct}}(\Delta \mathrm{Ct}=\mathrm{Ct}$ miR-451 - Ct U6). ATF2 expression was detected by immunohistochemistry. Staining was graded for intensity of staining ( 0 , negative; 1 , weak; 2 , moderate; 3 , strong) and percentage of cells stained. The combined staining score (extent $\mathrm{x}$ intensity) was reviewed independently by two investigators. (B) The cellular localization of ATF2 is shown. T, tumoral tissue; P, peritumoral tissue.

ATF2, miR-451 and ATF2 expression in the 26 pairs of tissues (including tumoral and peritumoral tissues from the 26 samples) was detected, which was represented by $2^{-\Delta \mathrm{Ct}}$ and ATF2 IHC score. Based on Spearman's rank analysis, an inverse correlation between miR-451 and ATF2 ( $\mathrm{r}=-0.5028$, $\mathrm{P}=0.0002$ ) was shown (Fig. 4A). Research has revealed that the 


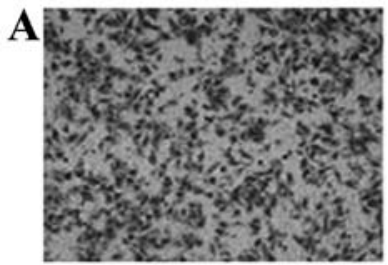

Mock

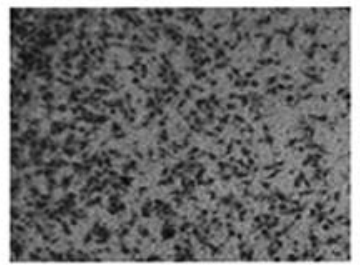

NC

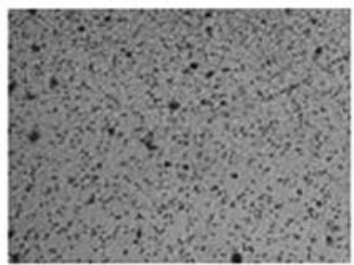

miR-451

B

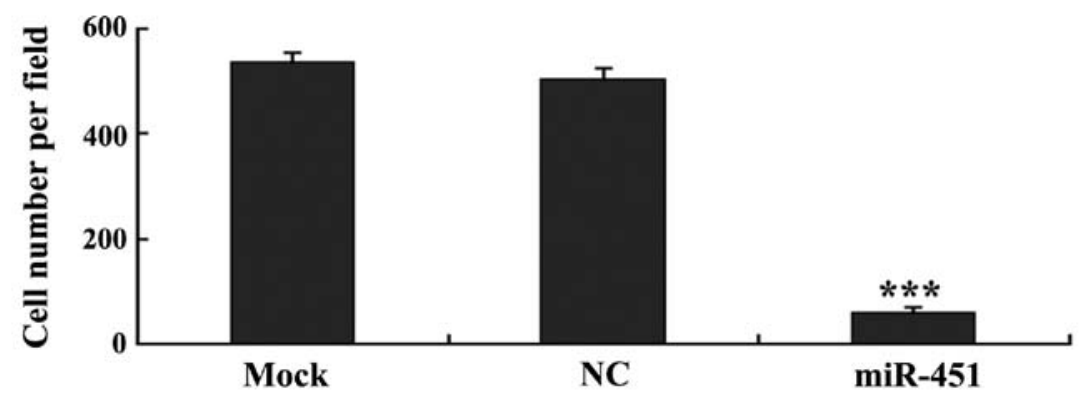

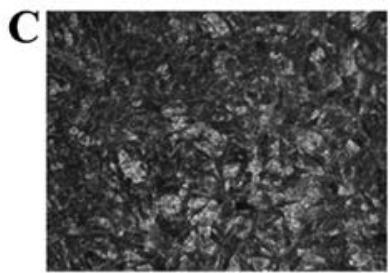

Mock

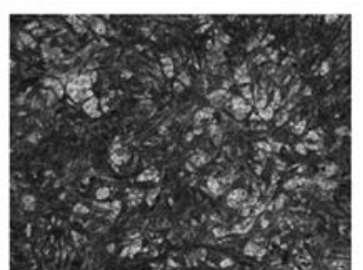

NC

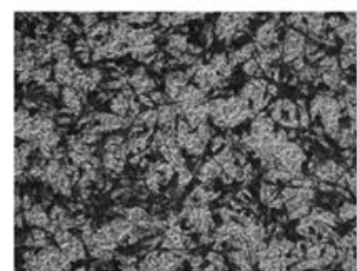

miR-451

Figure 5. miR-451 inhibits the migration of HepG2 and SK-Hep-1 cells. (A) Representative images of the HepG2 cell migration assay. (B) The cell numbers of HepG2 cells represent the average count of five random microscopic fields. Each bar represents the mean \pm standard deviation of the counts from a single representative experiment $\left({ }^{* * * *} \mathrm{P}<0.001\right)$. (C) Migration assay was performed using SK-Hep-1 cells.

location of ATF2 is related to its role in cancer (15). Further observation in this study showed that the upregulated ATF2 was mainly located in the nucleus of cells in the HCC tissues (Fig. 4B), which provides evidence that ATF2 has oncogenic activity in liver cancer. These results suggest that downregulation of miR-451 could contribute to the upregulation of ATF2 and the tumorigenesis of HCC.

miR-451 suppresses the migration of HepG2 and SK-Hep-1 cells. miR-451 has been reported to inhibit the migration of lung cancer cells (16). To evaluate its potential function in liver cancer, an in vitro transwell migration assay was performed using liver carcinoma cell lines, HepG2 and SK-Hep-1. The cells were cultured in DMEM containing 0.1\% FBS in the upper Transwell chamber and DMEM containing only 5\% FBS without cultured cells in the lower chamber. The data revealed that the numbers of HepG2 (Fig. 5A and B) and SK-Hep-1 (Fig. 5C) cells that migrated across the membrane were significantly reduced following transfection with miR-451 as compared to the number in the negative control cells. The results suggest that overexpression of miR-451 suppresses the migratory ability of liver carcinoma cells.

\section{Discussion}

In the present study, we have confirmed that miR-451 expression is downregulated in human HCCs and it is a direct negative regulator of ATF2 by targeting its $3^{\prime} \mathrm{UTR}$, which contributes to an increase in ATF2 in the nucleus of neoplastic tumor tissues inversely related to that of miR-451. Functional analysis revealed that miR-451 suppressed the migratory ability of hepatoma cell lines, HepG2 and SK-Hep-1. Thus, miR-451, as a suppressor, has an important role in the progression of hepatic carcinoma.

Dysregulated expression of miRNAs, that function as oncogenes or tumor suppressors, occurs in various types of cancers in a tissue-specific manner. However, widespread deregulation of miRNAs usually occurs in the same type of cancer. For example, in human HCC, miR-22 (17), let-7g (18), miR-29 (19), miR-124 and miR-199b-5p were found to be expressed at a lower level, indicating a poor survival or prognosis for patients with HCC. miR-451 has been reported to be highly conserved across vertebrates, and its biogenesis is independent of Dicer (20), mainly functioning as a tumor suppressor $(16,21)$, or acting to change the response to stress and start conditional control of cell proliferation and migration $(22,23)$. Suppression of miR-451 expression by tamoxifen was found to promote breast cancer cell survival and endocrine resistance (24), while transfection with the mature miR-451 was found to disperse neurospheres, and inhibit glioblastoma cell growth (25). In gastric cancer, miR-451 was also found to be decreased when compared to normal gastric mucosa (26). In the present study, we confirmed that miR-451 was markedly decreased in HCC and suppressed the migration of hepatoma 
cells; thus, it is considered as a potential tumor suppressor in HCC similar to its function in other tumors.

ATF2 may form homodimeric or heterodimeric activator protein 1 (AP1) which is involved in a series of eukaryotic cellular functions from cell proliferation and development to stress response and apoptosis $(26,27)$. ATF2 can elicit oncogene activities or tumor-suppressor activities depending on the cell or tissue type (28). For example, in melanoma, interfering with ATF2 transcriptional activity can inhibit the proliferation of melanoma cells in culture and the formation of tumors and metastasis (29,30). Contrarily, expression of transcriptionally inactive ATF2 in the presence of oncogene activation (such as Ras mutations) in non-melanoma skin cancers increases papilloma formation owing to the deregulated expression of genes that promote proliferation, such as CTNNB1 (31). Consistent with its cell cycle regulatory role, enhanced expression of ATF2 increased cell proliferation in mouse cancer models $(32,33)$.

However, how ATF2 elicits oncogenes or suppressors is not fully known. Increasing research suggests that subcellular localization of ATF2 is a crucial factor for its contrary activities. In melanomas, nuclear ATF2 is associated with metastasis and poor prognosis while cytoplasmic ATF2 is associated with nonmalignant skin cancers and more favorable prognosis (34). Its subcellular localization could be regulated by $\mathrm{PKC} \varepsilon$ that promotes oncogenic functions of ATF2 in the nucleus while blocking its apoptotic function in the mitochondria (15). In this research, we found that ATF2 was significantly increased in tumor tissue where it was localized in the nucleus in HCC compared to adjacent normal tissues. The detailed role of ATF2 in HCC requires further investigation. Choi et al noted that ATF2 can bind directly to HIF-1 $\alpha$ thereby competing with p53 leading to inhibition of the degradation of HIF-1 $\alpha$, and is essential for the full induction and maintaining the stability of HIF-1 $\alpha$ (35). A previous study confirmed that ATF2 is essential for hepatocyte survival in embryo development (27). Collectively, we speculate that ATF2 plays an oncogenic role in HCC.

Notably, ATF2 usually plays an important role as a transcriptional factor during response to stress. For example, transient elevation of ATF2 was observed in the MAPK pathway by hypoxia or low glucose. In contrast, to maintain survival and migration of tumor cells such as glioma, miR-451 downregulation is necessary at low energy (23). Thereby whether regulation by miR-451 of ATF2 is involved in cellular adaptation in response to cellular stress is a new topic which warrants further study.

Tumor invasion and metastasis are often associated with enhanced synthesis of matrix metalloproteinases (MMPs), and among these, MMP2 and MMP9 are of central importance. As an oncogene, ATF2 mediates MMP2 transcriptional activation induced by $\mathrm{p} 38$ in breast epithelial cells (36). If miR-451 could deregulate ATF2 expression, the migration of hepatoma cells could be inhibited. In the present study, miR-451 was found to inhibit the migration of hepatoma cell lines, HepG2 and SK-Hep-1.

In conclusion, miR-451 is a critical regulator of ATF2 in HCC and suppresses the migration of liver cancer cells. These findings suggest that miR-451 is a potential target for liver cancer therapy, and could be developed as a diagnostic or prognostic factor.

\section{Acknowledgements}

The present study was partially supported by the Chinese State Key Projects for Basic Research (nos. 2010CB912801 and 2009CB521804), and the Chinese National Natural Science Foundation Projects (nos. 81072021, 31170713 and 31270836).

\section{References}

1. Ferlay J, Shin HR, Bray F, Forman D, Mathers C and Parkin DM: Estimates of worldwide burden of cancer in 2008: GLOBOCAN 2008. Int J Cancer 127: 2893-917, 2010.

2. Sun H, Wei Y, Tu H, et al: Expressions of COX-2, PKC-alpha and miR-101 in gastric cancer and their correlations. Nan Fang Yi Ke Da Xue Xue Bao 33: 559-562, 2013 (In Chinese).

3. Bartel DP: MicroRNAs: genomics, biogenesis, mechanism, and function. Cell 116: 281-297, 2004.

4. Lujambio A and Lowe SW: The microcosmos of cancer. Nature 482: 347-355, 2012.

5. Augello C, Vaira V, Caruso L, et al: MicroRNA profiling of hepatocarcinogenesis identifies C19MC cluster as a novel prognostic biomarker in hepatocellular carcinoma. Liver Int 32: 772-782, 2012.

6. Ying Q, Liang L, Guo W, et al: Hypoxia-inducible microRNA-210 augments the metastatic potential of tumor cells by targeting vacuole membrane protein 1 in hepatocellular carcinoma. Hepatology 54: 2064-2075, 2011.

7. Fornari F, Gramantieri L, Giovannini C, et al: MiR-122/cyclin G1 interaction modulates p53 activity and affects doxorubicin sensitivity of human hepatocarcinoma cells. Cancer Res 69: 5761-5767, 2009.

8. Qu KZ, Zhang K, Li H, Afdhal NH and Albitar M: Circulating microRNAs as biomarkers for hepatocellular carcinoma. J Clin Gastroenterol 45: 355-360, 2011.

9. Tomimaru Y, Eguchi H, Nagano H, et al: Circulating microRNA-21 as a novel biomarker for hepatocellular carcinoma. J Hepatol 56: 167-175, 2012.

10. Liu Q, Fu H, Sun F, et al: miR-16 family induces cell cycle arrest by regulating multiple cell cycle genes. Nucleic Acids Res 36: 5391-5404, 2008.

11. Fu HJ, Zhu J, Yang M, et al: A novel method to monitor the expression of microRNAs. Mol Biotechnol 32: 197-204, 2006.

12. Bagga S, Bracht J, Hunter S, et al: Regulation by let-7 and lin-4 miRNAs results in target mRNA degradation. Cell 122: 553-563, 2005.

13. Lim LP, Lau NC, Garrett-Engele P, et al: Microarray analysis shows that some microRNAs downregulate large numbers of target mRNAs. Nature 433: 769-773, 2005.

14. Mathonnet G, Fabian MR, Svitkin YV, et al: MicroRNA inhibition of translation initiation in vitro by targeting the cap-binding complex eIF4F. Science 317: 1764-1767, 2007.

15. Lau E, Kluger H, Varsano T, et al: PKCepsilon promotes oncogenic functions of ATF2 in the nucleus while blocking its apoptotic function at mitochondria. Cell 148: 543-555, 2012.

16. Wang XC, Tian LL, Jiang XY, et al: The expression and function of miRNA-451 in non-small cell lung cancer. Cancer Lett 311: 203-209, 2011.

17. Zhang J, Yang Y, Yang T, et al: microRNA-22, downregulated in hepatocellular carcinoma and correlated with prognosis, suppresses cell proliferation and tumourigenicity. $\mathrm{Br} \mathrm{J}$ Cancer 103: 1215-1220, 2010.

18. Ji J, Zhao L, Budhu A, et al: Let-7g targets collagen type I alpha2 and inhibits cell migration in hepatocellular carcinoma. J Hepatol 52: 690-697, 2010.

19. Xiong Y, Fang JH, Yun JP, et al: Effects of microRNA-29 on apoptosis, tumorigenicity, and prognosis of hepatocellular carcinoma. Hepatology 51: 836-845, 2010.

20. Yang JS, Maurin T, Robine N, et al: Conserved vertebrate mir-451 provides a platform for Dicer-independent, Ago2mediated microRNA biogenesis. Proc Natl Acad Sci USA 107: 15163-15168, 2010.

21. Wang R, Wang ZX, Yang JS, Pan X, De W and Chen LB: MicroRNA-451 functions as a tumor suppressor in human non-small cell lung cancer by targeting ras-related protein 14 (RAB14). Oncogene 30: 2644-2658, 2011.

22. Godlewski J, Nowicki MO, Bronisz A, et al: MicroRNA-451 regulates LKB1/AMPK signaling and allows adaptation to metabolic stress in glioma cells. Mol Cell 37: 620-632, 2010. 
23. Godlewski J, Bronisz A, Nowicki MO, Chiocca EA and Lawler S microRNA-451: A conditional switch controlling glioma cell proliferation and migration. Cell Cycle 9: 2742-2748, 2010.

24. Bergamaschi A and Katzenellenbogen BS: Tamoxifen downregulation of miR-451 increases $14-3-3 \zeta$ and promotes breast cancer cell survival and endocrine resistance. Oncogene 31 : 39-47, 2012.

25. Gal H, Pandi G, Kanner AA, et al: miR-451 and imatinib mesylate inhibit tumor growth of glioblastoma stem cells Biochem Biophys Res Commun 376: 86-90, 2008.

26. Hai $\mathrm{T}$ and Hartman MG: The molecular biology and nomenclature of the activating transcription factor/cAMP responsive element binding family of transcription factors: activating transcription factor proteins and homeostasis. Gene 273: 1-11, 2001.

27. Breitwieser W, Lyons S, Flenniken AM, et al: Feedback regulation of p38 activity via ATF2 is essential for survival of embryonic liver cells. Genes Dev 21: 2069-2082, 2007.

28. Eferl R and Wagner EF: AP-1: a double-edged sword in tumorigenesis. Nat Rev Cancer 3: 859-868, 2003.

29. Bhoumik A, Gangi L and Ronai Z: Inhibition of melanoma growth and metastasis by ATF2-derived peptides. Cancer Res 64: 8222-8230, 2004.

30. Bhoumik A, Huang TG, Ivanov V, et al: An ATF2-derived peptide sensitizes melanomas to apoptosis and inhibits their growth and metastasis. J Clin Invest 110: 643-650, 2002.
31. Bhoumik A, Fichtman B, Derossi C, et al: Suppressor role of activating transcription factor 2 (ATF2) in skin cancer. Proc Natl Acad Sci USA 105: 1674-1679, 2008

32. Vale-Cruz DS, Ma Q, Syme J and LuValle PA: Activating transcription factor- 2 affects skeletal growth by modulating $\mathrm{pRb}$ gene expression. Mech Dev 125: 843-856, 2008.

33. Nakamura T, Okuyama S, Okamoto S, Nakajima T, Sekiya S and Oda K: Down-regulation of the cyclin A promoter in differentiating human embryonal carcinoma cells is mediated by depletion of ATF-1 and ATF-2 in the complex at the ATF/CRE site. Exp Cell Res 216: 422-430, 1995.

34. Berger AJ, Kluger HM, Li N, et al: Subcellular localization of activating transcription factor 2 in melanoma specimens predicts patient survival. Cancer Res 63: 8103-8107, 2003.

35. Choi JH, Cho HK, Choi YH and Cheong J: Activating transcription factor 2 increases transactivation and protein stability of hypoxia-inducible factor 1alpha in hepatocytes. Biochem J 424: 285-296, 2009.

36. Song H, Ki SH, Kim SG and Moon A: Activating transcription factor 2 mediates matrix metalloproteinase- 2 transcriptional activation induced by p38 in breast epithelial cells. Cancer Res 66: 10487-10496, 2006. 